\title{
Normal values and associated factors in high- resolution anorectal manometry in healthy Vietnamese adults
}

Nguyen Ngoc Anh ( $\square$ nngocanh@hmu.edu.vn )

Hanoi Medical University https://orcid.org/0000-0001-7383-0652

Le Dinh Tung

Hanoi Medical University

Ngo Xuan Khoa

Hanoi Medical University

Le Manh Cuong

National Hospital of Traditional Medicine

Nguyen Xuan Hung

Viet Duc Hospital

Nguyen Thi Thu Hang

Viet Duc Hospital

Pham Phuc Khanh

Viet Duc hospital

Nguyen Van Huy

St. Luke's International University

Vu Duy Kien

Oncare Medical Technology Company Limited

Research article

Keywords: High-resolution anorectal manometry, Anorectal manometry, Normal Values, Healthy people, Vietnam

Posted Date: July 20th, 2020

DOI: https://doi.org/10.21203/rs.3.rs-42055/v1

License: () (1) This work is licensed under a Creative Commons Attribution 4.0 International License.

Read Full License 


\section{Abstract \\ Background}

This study aims to identify normal HRAM values and related factors in healthy Vietnamese adults.

\section{Methods}

This cross-sectional study was conducted at Viet Duc hospital, Hanoi, Vietnam, during April and May, 2019. Healthy volunteers were recruited to participate in the study. Anorectal measurement values including pressure and an electromyographical signal from the digestive tract were recorded. Differences between groups were analyzed using paired t-tests, and linear regression models was used to compare anorectal values between men and women.

\section{Results}

A total of the 76 healthy volunteers was recruited. The mean functional anal canal length was $4.2 \pm$ $0.5 \mathrm{~cm}$ while the mean anal high-pressure zone (HPZ) length was $3.4 \pm 0.5 \mathrm{~cm}$. The mean defecation index was $1.4 \pm 0.8$, with values ranging from 0.3 to 5.0 . The mean threshold volume to elicit RAIR was $18.1 \mathrm{~mL}$. Mean rectal sensation values were $32.4 \mathrm{~mL}, 81.6 \mathrm{~mL}$, and $159 \mathrm{~mL}$ at the first sensation, the desire to defecate, and the urge to defecate, respectively. Dyssynergic patterns occurred in approximately $50 \%$ of study participants and included mainly types I (27.6\%) and III (14.6\%). There were significant differences between males and females in maximum anal squeeze pressure, maximum anal cough pressure, maximum anal strain pressure, maximum rectal cough pressure, and maximum rectal strain pressure (all $p<0.01)$.

\section{Conclusions}

This study establishes normal HRAM values in healthy Vietnamese adults, particularly with regards to normal values of anorectal pressure and rectal sensation. Further studies that include larger sample sizes should be conducted in order to further confirm the constants and their relationships.

\section{Background}

Anorectal manometry (ARM) is a primary method for evaluating anorectal function [1, 2]. While conventional ARM has been used to effectively diagnose and manage anorectal disorders [3-5], that technique has limitations that affect the measurement and interpretation of results $[6,7]$. In response to these limitations, high-resolution anorectal manometry (HRAM) has been developed over the past ten years and has been shown to provide advantages in comparison to conventional ARM [2]. Among these, the most advantageous aspects of HRAM include improvements to sensor systems and the computer 
software used to record and replicate ARM values over time and anorectal locations. The HRAM uses the technique of stationary examination to provide detailed topographic and colorimetric mapping of the anorectal area [2]. In order to standardize the HRAM technique, it is necessary to identify normal values that can be used as a baseline for comparison with specific disease cases. Knowledge of normal HRAM values thus enhances our understanding of anorectal disorder-related diseases.

Studies regarding normal HRAM values in healthy people have been conducted in several countries [811]. In Vietnam, a single study using HRAM has been conducted, which reported values in patients with hemorrhoids before and after doppler-guided transanal hemorrhoidal dearterialization [12]. Although use of HRAM has become widespread, there are currently no reports of normal HRAM values in Vietnam. Therefore, the aim of this study is to identify normal HRAM values and related factors in healthy Vietnamese adults.

\section{Methods}

This cross-sectional study was conducted at Viet Duc hospital, Hanoi, Vietnam, during April and May, 2019. This study received ethical approval from the ethics committee of the Hanoi Medical University, Hanoi, Vietnam (ref. 21NCS17/HDDDDHYHN; dated 08/02/2018). Healthy volunteers were recruited to participate in the study and study aims, objectives, possible adverse effects of anorectal HRAM, and the right to withdraw from the study at any time without consequences were explained to them. All participants provided written consent before participating in the study.

We calculated the sample size using the formula for estimating a population mean proposed by the World Health Organization using a significance level of 0.05 [13]. We assumed a maximum resting pressure of $69.1 \mathrm{~mm} \mathrm{Hg}$ according to values reported by Li et al. [9]. We assumed a population standard deviation of the maximum resting pressure of $15 \mathrm{~mm} \mathrm{Hg}$ and a relative precision of $5 \%$. After controlling for an estimated $5 \%$ non-response rate, we calculated a minimum sample size of 76 patients.

Inclusion criteria for participants included age of 18 to 75 years, normal defecation for the three months prior to enrollment, and normal physical abdominal and digital anorectal examinations. Exclusion criteria included constipation, fecal incontinence, obstructed defecation, hematochezia, hemorrhoid, rectal mucus discharge, and any history of anorectal diseases. We excluded pregnant women, people with cardiovascular, respiratory, psychiatric, neurologic, endocrine, hepatic, or renal diseases. Digital rectal examination was performed in all participants to ensure they were free from any anorectal diseases. We also collected relevant patient data such as sex, age, height, and weight.

Anorectal manometry was performed using the ISOLAB high-resolution manometry system (Standard Instruments $\mathrm{GmbH}$, Karlsruhe, Germany) which included a $6 \mathrm{~mm}$ diameter catheter probe possessing eight channel sensors. Anorectal measurement values including pressure and an electromyographical signal from the digestive tract were recorded. We used the ViMeDat (Visible Medical Data; Standard Instruments $\mathrm{GmbH}$, Karlsruhe, Germany) software to extract values after measurement. Bowel preparation included a Fleet enema (C.B. Fleet Company, Inc., Lynchburg, VA, USA) or patient defecation, if 
possible, approximately 2 hours before the measurement. All measurements were taken with study participants placed in the left-lateral position with knees and hips flexed at an angle of ninety degrees. The catheter probe was lubricated and gently inserted into the rectal ampulla about $6 \mathrm{~cm}$ from the anal verge while maintaining the most proximal sensor of the catheter probe outside of the anal canal. We assessed anorectal parameters during periods of resting, squeezing, pushing, and coughing. During the resting period, the participant relaxed by lying still and not speaking for approximately 5 minutes. During the squeezing period, the participant was instructed to squeeze the anal canal as tightly as possible for approximately 20 seconds. During the pushing period, the participant was asked to bear down, as if to defecate, for approximately 10-20 seconds. During the coughing period, the participant was asked to cough five to seven times. Measurements for each period type were gathered twice.

We detected the recto-anal inhibitory reflex (RAIR) by injecting (with $10 \mathrm{~mL}$ increments) up to $50 \mathrm{~mL}$ of air into a rectal balloon that was attached to the head of the catheter probe. In this study, we assessed if the RAIR present or absent, with RAIR was considered present if anal relaxation was greater than $25 \%$ [14]. We recorded the threshold volume in $\mathrm{mL}$ of air required to elicit RAIR. We also used the rectal balloon to measure rectal sensation. The rectal balloon was distended in $10 \mathrm{~mL}$ increments up to $300 \mathrm{~mL}$ of air, and the participants were instructed to describe their sensations as first sensation, desire to defecate, and urge to defecate. In this study, we defined the defecation index as the ratio of the maximum rectal strain pressure to the minimum anal strain pressure. We used the classification system proposed by Rao et al. to define dyssynergic patterns during the period of attempted defecation, including types I-IV, where Type I was considered a rise in rectal pressure $\geq 40 \mathrm{~mm} \mathrm{Hg}$ with an increase in anal pressure, Type II was considered a rise in rectal pressure $<40 \mathrm{~mm} \mathrm{Hg}$ with an increase in anal pressure, Type III was considered a rise in rectal pressure $\geq 40 \mathrm{~mm} \mathrm{Hg}$ and with an absent relaxation or inadequate $(\leq 20 \%)$ relaxation of the anal sphincter, and Type IV included no rise in rectal pressure and an absent relaxation or inadequate relaxation of the anal sphincter [15].

Analytical results of quantitative variables were presented as means and standard deviations whereas those of qualitative variables were presented as absolute values and percentages. Differences between groups were analyzed using paired t-tests. We also compared anorectal manometric measurements between men and women using three multivariate linear regression models, including Model 1 (by sex, adjusted by age), Model 2 (by sex, adjusted by BMI), and Model 3 (by sex, adjusted by both age and BMI). All statistical analyses were conducted using STATA 14.0 (Stata Corp, College Station, TX, USA). Statistical levels were considered significant at $p$-values $<0.05$.

\section{Results}

A total of the 76 healthy volunteers was recruited (age range: 21-73 years), which included 38 males (age range: $24-73$ years) and 38 females (age range: $21-68$ years). Table 1 shows the general characteristics of the study participants. The mean age of participants was $41.5 \pm 13.3$ years. The proportion of study participants 40 years old or younger was higher among females $(65.8 \%)$ than among males $(52.6 \%)$, but this difference was not statistically significant $(p=0.24)$. The mean BMI was $22.4 \pm 2.5$, and was 
significantly higher among males than among females $(23.2 \pm 2.7$ vs $21.7 \pm 2.1, p<0.01)$. Only $14.5 \%$ of people were overweight $(\mathrm{BMI} \geq 25)$, a proportion that was higher among males than among females, although the difference was not statistically significant $(p=0.19)$.

Table 1

Characteristics of the 76 healthy study participants

\begin{tabular}{|c|c|c|c|c|}
\hline & Total $(n=76)$ & Male $(n=38)$ & Female $(n=38)$ & p-value \\
\hline & \multicolumn{3}{|c|}{$\mathrm{N}(\%)$ or mean (SD) } & \\
\hline Age (years) & $41.5(13.3)$ & $40.5(13.4)$ & $42.6(13.2)$ & 0.49 \\
\hline \multicolumn{5}{|c|}{ Age Group (years) } \\
\hline$\leq 40$ & $45(59.2)$ & $20(52.6)$ & $25(65.8)$ & \multirow[t]{2}{*}{0.24} \\
\hline$>40$ & $31(40.8)$ & $18(47.4)$ & $13(34.2)$ & \\
\hline BMI & $22.4(2.5)$ & $23.2(2.7)$ & $21.7(2.1)$ & $<0.01$ \\
\hline \multicolumn{5}{|l|}{ BMI } \\
\hline$<25$ & $65(85.5)$ & $30(79.8)$ & $35(92.1)$ & \multirow[t]{2}{*}{0.19} \\
\hline$\geq 25$ & $11(14.5)$ & $8(21.2)$ & $3(7.9)$ & \\
\hline
\end{tabular}

Table 2 presents HRAM values in healthy participants. The mean functional anal canal length was $4.2 \pm$ $0.5 \mathrm{~cm}$ while the mean anal high-pressure zone (HPZ) length was $3.4 \pm 0.5 \mathrm{~cm}$. Within the anal pressure parameter values, the mean maximum resting pressure was the lowest, at $71.5 \pm 17.4 \mathrm{~mm} \mathrm{Hg}$, whereas the mean maximum squeeze pressure was the highest, at $163.3 \pm 59.1 \mathrm{~mm} \mathrm{Hg}$. For the rectal pressure parameter values, the mean maximum cough pressure was higher than the mean maximum strain pressure. The mean defecation index was $1.4 \pm 0.8$, with values ranging from 0.3 to 5.0 . The mean threshold volume to elicit RAIR was $18.1 \mathrm{~mL}$. Mean rectal sensation values were $32.4 \mathrm{~mL}, 81.6 \mathrm{~mL}$, and $159.0 \mathrm{~mL}$ at the first sensation, the desire to defecate, and the urge to defecate, respectively. Dyssynergic patterns occurred in approximately $50 \%$ of study participants and included mainly types I $(27.6 \%)$ and III (14.6\%). 
Table 2

High-resolution anorectal manometry (HRAM) parameters in 76 healthy study participants $\mathrm{N}(\%)$ or mean (SD)

Min-Max

\begin{tabular}{|c|c|c|}
\hline Functional anal canal length $(\mathrm{cm})$ & $4.2(0.5)$ & $3.2-4.8$ \\
\hline HPZ length $(\mathrm{cm})$ & $3.4(0.5)$ & $2.4-4$ \\
\hline \multicolumn{3}{|l|}{ Anal pressure } \\
\hline Maximum resting pressure $(\mathrm{mm} \mathrm{Hg})$ & $71.5(17.4)$ & $31.3-115.9$ \\
\hline Maximum squeeze pressure $(\mathrm{mm} \mathrm{Hg})$ & $163.3(59.1)$ & $52.2-355.5$ \\
\hline Maximum cough pressure $(\mathrm{mm} \mathrm{Hg})$ & $99.5(31.2)$ & $38.4-191.5$ \\
\hline Maximum strain pressure $(\mathrm{mm} \mathrm{Hg})$ & $74.7(28.9)$ & 23.4-169.1 \\
\hline \multicolumn{3}{|l|}{ Rectal pressure } \\
\hline Maximum cough pressure $(\mathrm{mm} \mathrm{Hg})$ & $89.8(35.1)$ & $36.1-197.3$ \\
\hline Maximum strain pressure $(\mathrm{mm} \mathrm{Hg})$ & $63.1(26.3)$ & 18.8-163.1 \\
\hline Defecation index & $1.4(0.8)$ & $0.3-5$ \\
\hline Threshold volume to elicit RAIR (ml) & $18.1(8.3)$ & $10-50$ \\
\hline \multicolumn{3}{|l|}{ Rectal sensation } \\
\hline First sensation (mL) & $32.4(10.9)$ & $20-60$ \\
\hline Desire to defecate $(\mathrm{mL})$ & $81.6(19.3)$ & $50-120$ \\
\hline Urge to defecate $(\mathrm{mL})$ & $159.9(31.5)$ & $100-240$ \\
\hline \multicolumn{3}{|l|}{ Dyssynergic pattern } \\
\hline Normal & $38(50.0)$ & NA \\
\hline Type I & $21(27.6)$ & NA \\
\hline Type II & $3(3.9)$ & NA \\
\hline Type III & $11(14.6)$ & NA \\
\hline Type IV & $3(3.9)$ & NA \\
\hline
\end{tabular}

List of abbreviations: SD: Standard Deviation; HPZ: High-Pressure Zone, RAIR: Rectoanal Inhibitory Reflex, NA: Not Applicable

HRAM values are presented in Table 3 according to participant sex, age group, and BMI group. There were significant differences between males and females in the means of maximum anal squeeze pressure, maximum anal cough pressure, maximum anal strain pressure, maximum rectal cough pressure, and maximum rectal strain pressure (all $p<0.01$ ). Regarding age, mean maximum rectal cough pressure and 
maximum rectal strain pressure were significantly higher in people aged greater than 40 years than in those aged 40 years or less (both $p<0.05$ ). The threshold volume to elicit RAIR among people aged above 40 years was higher than that among people aged 40 years or less $(p=0.02)$. The volume at first sensation and desire to defecate were higher among people aged above 40 as compared to those aged 40 or less (both $p<0.01$ ). The length of both the functional anal canal and the HPZ were significantly greater in people with a BMI of 25 or higher than in people with a BMI of less than 25 (both $p<0.01$ ). Anal pressure values tended to be higher in people with a BMI of 25 or greater than in people with BMI less than 25 , but the difference was significant only with the maximum anal strain pressure $(p=0.01)$. Furthermore, difference in rectal sensation between people with a BMI of 25 or higher and those with a BMI lower than 25 were significant at the rectal first sensation $(p=0.01)$ but not at the other two rectal sensation parameters. Finally, linear regressions were performed in order to assess the effect of sex (adjusted for age, BMI, or both age and BMI) on the various HRAM parameters (Table 4). Significant differences in mean maximum anal squeeze pressure, maximum anal cough pressure, maximum anal strain pressure, maximum rectal cough pressure, and maximum rectal strain pressure between male and female participants were recorded (all $p<0.05$ ). 
Table 3

High-resolution anorectal manometry (HRAM) parameters in 76 healthy participants according to sex, age group, and BMI group

\begin{tabular}{|c|c|c|c|c|c|c|c|c|c|}
\hline \multicolumn{4}{|c|}{ Sex } & \multicolumn{3}{|c|}{ Age group } & \multicolumn{3}{|c|}{ BMI group } \\
\hline $\begin{array}{l}\text { Male } \\
(n=38)\end{array}$ & $\begin{array}{l}\text { Female } \\
(\mathrm{n}= \\
38)\end{array}$ & $\begin{array}{l}\mathrm{p}- \\
\text { value }\end{array}$ & $\begin{array}{l}\text { Age } \\
\leq 40 \\
(n= \\
45)\end{array}$ & $\begin{array}{l}\text { Age > } \\
40(n \\
=31)\end{array}$ & $\begin{array}{l}\mathrm{p}- \\
\text { value }\end{array}$ & $\begin{array}{l}\text { BMI } \\
<25 \\
(n= \\
65)\end{array}$ & $\begin{array}{l}\mathrm{BMI} \geq 25 \\
(\mathrm{n}=11)\end{array}$ & $\mathrm{p}$-value & \\
\hline \multicolumn{3}{|c|}{$\mathrm{N}(\%)$ or mean (SD) } & \multicolumn{3}{|c|}{$\begin{array}{l}\mathrm{N}(\%) \text { or mean } \\
(\mathrm{SD})\end{array}$} & \multicolumn{4}{|c|}{$\begin{array}{l}\mathrm{N}(\%) \text { or mean } \\
(\mathrm{SD})\end{array}$} \\
\hline $\begin{array}{l}\text { Functional } \\
\text { anal canal } \\
\text { length (cm) }\end{array}$ & $\begin{array}{l}4.3 \\
(0.5)\end{array}$ & $\begin{array}{l}4.1 \\
(0.5)\end{array}$ & 0.09 & $\begin{array}{l}4.2 \\
(0.5)\end{array}$ & $\begin{array}{l}4.3 \\
(0.5)\end{array}$ & 0.40 & $4.1(0.5)$ & $\begin{array}{l}4.7 \\
(0.3)\end{array}$ & $<.01$ \\
\hline $\begin{array}{l}\text { HPZ length } \\
(\mathrm{cm})\end{array}$ & $\begin{array}{l}3.5 \\
(0.5)\end{array}$ & $\begin{array}{l}3.3 \\
(0.5)\end{array}$ & 0.09 & $\begin{array}{l}3.4 \\
(0.5)\end{array}$ & $\begin{array}{l}3.5 \\
(0.5)\end{array}$ & 0.40 & $3.3(0.5)$ & $\begin{array}{l}3.9 \\
(0.3)\end{array}$ & $<.01$ \\
\hline \multicolumn{10}{|l|}{ Anal pressure } \\
\hline $\begin{array}{l}\text { Maximum } \\
\text { resting } \\
\text { pressure (mm } \\
\mathrm{Hg})\end{array}$ & $\begin{array}{l}74.9 \\
(16.3)\end{array}$ & $\begin{array}{l}68.0 \\
(18.0)\end{array}$ & 0.08 & $\begin{array}{l}73.7 \\
(17.9)\end{array}$ & $\begin{array}{l}68.2 \\
(16.4)\end{array}$ & 0.18 & $\begin{array}{l}70.3 \\
(18.4)\end{array}$ & $\begin{array}{l}78.1 \\
(7.8)\end{array}$ & 0.17 \\
\hline $\begin{array}{l}\text { Maximum } \\
\text { squeeze } \\
\text { pressure (mm } \\
\mathrm{Hg})\end{array}$ & $\begin{array}{l}183.3 \\
(48.0)\end{array}$ & $\begin{array}{l}143.3 \\
(62.0)\end{array}$ & $<.01$ & $\begin{array}{l}159.3 \\
(60.1)\end{array}$ & $\begin{array}{l}169.0 \\
(58.1)\end{array}$ & 0.48 & $\begin{array}{l}161.2 \\
(58.5)\end{array}$ & $\begin{array}{l}175.4 \\
(64.6)\end{array}$ & 0.47 \\
\hline $\begin{array}{l}\text { Maximum } \\
\text { cough } \\
\text { pressure (mm } \\
\mathrm{Hg})\end{array}$ & $\begin{array}{l}112.2 \\
(31.9)\end{array}$ & $\begin{array}{l}86.7 \\
(24.8)\end{array}$ & $<0.01$ & $\begin{array}{l}99.7 \\
(29.4)\end{array}$ & $\begin{array}{l}99.2 \\
(34.0)\end{array}$ & 0.94 & $\begin{array}{l}97.5 \\
(30.1)\end{array}$ & $\begin{array}{l}111.0 \\
(36.2)\end{array}$ & 0.19 \\
\hline $\begin{array}{l}\text { Maximum } \\
\text { strain pressure } \\
(\mathrm{mm} \mathrm{Hg})\end{array}$ & $\begin{array}{l}84.1 \\
(28.4)\end{array}$ & $\begin{array}{l}65.4 \\
(26.7)\end{array}$ & $<01$ & $\begin{array}{l}73.9 \\
(28.9)\end{array}$ & $\begin{array}{l}76.0 \\
(29.4)\end{array}$ & 0.76 & $\begin{array}{l}71.3 \\
(28.9)\end{array}$ & $\begin{array}{l}95.2 \\
(20.3)\end{array}$ & 0.01 \\
\hline \multicolumn{10}{|l|}{$\begin{array}{l}\text { Rectal } \\
\text { pressure }\end{array}$} \\
\hline $\begin{array}{l}\text { Maximum } \\
\text { cough } \\
\text { pressure (mm } \\
\mathrm{Hg})\end{array}$ & $\begin{array}{l}101.7 \\
(38.7)\end{array}$ & $\begin{array}{l}78.0 \\
(26.7)\end{array}$ & 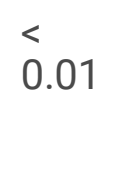 & $\begin{array}{l}81.4 \\
(34.2)\end{array}$ & $\begin{array}{l}102.1 \\
(33.2)\end{array}$ & 0.01 & $\begin{array}{l}87.5 \\
(34.2)\end{array}$ & $\begin{array}{l}103.5 \\
(39.3)\end{array}$ & 0.17 \\
\hline $\begin{array}{l}\text { Maximum } \\
\text { strain pressure } \\
(\mathrm{mm} \mathrm{Hg})\end{array}$ & $\begin{array}{l}75.1 \\
(27.6)\end{array}$ & $\begin{array}{l}51.0 \\
(18.5)\end{array}$ & $<0.01$ & $\begin{array}{l}58.1 \\
(24.4)\end{array}$ & $\begin{array}{l}70.3 \\
(27.7)\end{array}$ & 0.04 & $\begin{array}{l}61.3 \\
(25.3)\end{array}$ & $\begin{array}{l}73.8 \\
(31.1)\end{array}$ & 0.15 \\
\hline $\begin{array}{l}\text { Defecation } \\
\text { index }\end{array}$ & $\begin{array}{l}1.4 \\
(0.7)\end{array}$ & $\begin{array}{l}1.4 \\
(0.8)\end{array}$ & 0.93 & $\begin{array}{l}1.4 \\
(0.8)\end{array}$ & $\begin{array}{l}1.4 \\
(0.7)\end{array}$ & 0.92 & $1.4(0.8)$ & $\begin{array}{l}1.1 \\
(0.5)\end{array}$ & 0.28 \\
\hline
\end{tabular}




\begin{tabular}{|c|c|c|c|c|c|c|c|c|c|}
\hline & Sex & & & Age gro & & & $\mathrm{BMI}$ grc & & \\
\hline $\begin{array}{l}\text { Threshold } \\
\text { volume to elicit } \\
\text { RAIR (mL) }\end{array}$ & $\begin{array}{l}18.7 \\
(9.1)\end{array}$ & $\begin{array}{l}17.5 \\
(7.5)\end{array}$ & 0.53 & $\begin{array}{l}16.3 \\
(8.6)\end{array}$ & $\begin{array}{l}20.6 \\
(7.3)\end{array}$ & 0.02 & $\begin{array}{l}18.1 \\
(8.5)\end{array}$ & $\begin{array}{l}18.2 \\
(7.5)\end{array}$ & 0.97 \\
\hline $\begin{array}{l}\text { Rectal } \\
\text { sensation }\end{array}$ & & & & & & & & & \\
\hline $\begin{array}{l}\text { First sensation } \\
(\mathrm{mL})\end{array}$ & $\begin{array}{l}32.6 \\
(10.6)\end{array}$ & $\begin{array}{l}32.1 \\
(11.4)\end{array}$ & 0.83 & $\begin{array}{l}28.4 \\
(7.1)\end{array}$ & $\begin{array}{l}38.1 \\
(13.0)\end{array}$ & $\stackrel{<}{0.01}$ & $\begin{array}{l}31.2 \\
(10.4)\end{array}$ & $\begin{array}{l}39.1 \\
(12.2)\end{array}$ & 0.03 \\
\hline $\begin{array}{l}\text { Desire to } \\
\text { defecate }(\mathrm{mL})\end{array}$ & $\begin{array}{l}79.2 \\
(18.4)\end{array}$ & $\begin{array}{l}83.9 \\
(20.2)\end{array}$ & 0.29 & $\begin{array}{l}76.7 \\
(17.6)\end{array}$ & $\begin{array}{l}88.7 \\
(19.8)\end{array}$ & $<.01$ & $\begin{array}{l}80.6 \\
(18.9)\end{array}$ & $\begin{array}{l}87.3 \\
(21.5)\end{array}$ & 0.29 \\
\hline $\begin{array}{l}\text { Urge to } \\
\text { defecate }(\mathrm{mL})\end{array}$ & $\begin{array}{l}159.2 \\
(30.6)\end{array}$ & $\begin{array}{l}160.5 \\
(32.7)\end{array}$ & 0.86 & $\begin{array}{l}156.9 \\
(32.6)\end{array}$ & $\begin{array}{l}164.2 \\
(29.8)\end{array}$ & 0.32 & $\begin{array}{l}159.2 \\
(30.6)\end{array}$ & $\begin{array}{l}163.6 \\
(35.6)\end{array}$ & 0.67 \\
\hline $\begin{array}{l}\text { Dyssynergic } \\
\text { pattern- } \mathrm{N}(\%)\end{array}$ & & & & & & & & & \\
\hline Normal & $\begin{array}{l}16 \\
(42.1)\end{array}$ & $\begin{array}{l}22 \\
(57.9)\end{array}$ & 0.10 & $\begin{array}{l}22 \\
(48.9)\end{array}$ & $\begin{array}{l}16 \\
(51.6)\end{array}$ & 1.00 & $\begin{array}{l}34 \\
(52.3)\end{array}$ & $\begin{array}{l}4 \\
(36.4)\end{array}$ & 0.43 \\
\hline Type I & $\begin{array}{l}14 \\
(36.8)\end{array}$ & $\begin{array}{l}7 \\
(18.4)\end{array}$ & & $\begin{array}{l}12 \\
(26.7)\end{array}$ & $\begin{array}{l}9 \\
(29.0)\end{array}$ & & $\begin{array}{l}16 \\
(24.6)\end{array}$ & $\begin{array}{l}5 \\
(45.4)\end{array}$ & \\
\hline Type II & $0(0.0)$ & $\begin{array}{l}3 \\
(7.9)\end{array}$ & & $2(4.4)$ & $\begin{array}{l}1 \\
(3.2)\end{array}$ & & $2(3.1)$ & $\begin{array}{l}1 \\
(9.1)\end{array}$ & \\
\hline Type III & $\begin{array}{l}7 \\
(18.5)\end{array}$ & $\begin{array}{l}4 \\
(10.5)\end{array}$ & & $\begin{array}{l}7 \\
(15.6)\end{array}$ & $\begin{array}{l}4 \\
(12.9)\end{array}$ & & $\begin{array}{l}10 \\
(15.4)\end{array}$ & $\begin{array}{l}1 \\
(9.1)\end{array}$ & \\
\hline Type IV & $1(2.6)$ & $\begin{array}{l}2 \\
(5.3)\end{array}$ & & $2(4.4)$ & $\begin{array}{l}1 \\
\text { (3.3) }\end{array}$ & & $3(4.6)$ & $\begin{array}{l}0 \\
(0.0)\end{array}$ & \\
\hline
\end{tabular}

List of abbreviations: SD: Standard Deviation; HPZ: High-Pressure Zone; RAIR: Rectoanal Inhibitory Reflex; BMI: Body Mass Index 
Table 4

Multiple linear regression analyses of various high-resolution anorectal manometry (HRAM) parameters for sex (ref.: female) according to various models

\begin{tabular}{|c|c|c|c|c|c|c|}
\hline & Model 1 & & Model 2 & & Model 3 & \\
\hline & $\beta(95 \% \mathrm{Cl})$ & $\begin{array}{l}\mathrm{p}- \\
\text { value }\end{array}$ & $\beta(95 \% \mathrm{Cl})$ & $\begin{array}{l}\mathrm{p}- \\
\text { value }\end{array}$ & $\beta(95 \% \mathrm{Cl})$ & $\begin{array}{l}\mathrm{p}- \\
\text { value }\end{array}$ \\
\hline $\begin{array}{l}\text { Functional anal canal length } \\
(\mathrm{cm})\end{array}$ & $\begin{array}{l}0.20(-0.02 \\
0.42)\end{array}$ & 0.08 & $\begin{array}{l}0.12 \\
(-0.11,0.34)\end{array}$ & 0.31 & $\begin{array}{l}0.12(-0.10 \\
0.36)\end{array}$ & 0.27 \\
\hline HPZ length (cm) & $\begin{array}{l}0.20(-0.02 \\
0.42)\end{array}$ & 0.08 & $\begin{array}{l}0.12 \\
(-0.11,0.34)\end{array}$ & 0.31 & $\begin{array}{l}0.13(-0.10 \\
0.36)\end{array}$ & 0.27 \\
\hline \multicolumn{7}{|l|}{ Anal pressure } \\
\hline $\begin{array}{l}\text { Maximum resting pressure } \\
(\mathrm{mm} \mathrm{Hg})\end{array}$ & $\begin{array}{l}6.44(-1.35 \\
14.24)\end{array}$ & 0.10 & $\begin{array}{l}6.58(-1.69 \\
14.86)\end{array}$ & 0.12 & $\begin{array}{l}5.43(-2.84 \\
13.70)\end{array}$ & 0.19 \\
\hline $\begin{array}{l}\text { Maximum squeeze pressure } \\
(\mathrm{mm} \mathrm{Hg})\end{array}$ & $\begin{array}{l}40.56 \\
(14.75 \\
66,37)\end{array}$ & $<.01$ & $\begin{array}{l}40.32 \\
(13.31 \\
67.32)\end{array}$ & $\begin{array}{l}<.01 \\
0.01\end{array}$ & $\begin{array}{l}41.47 \\
(13.98 \\
68.96)\end{array}$ & $\dot{0} 01$ \\
\hline $\begin{array}{l}\text { Maximum cough pressure } \\
(\mathrm{mm} \mathrm{Hg})\end{array}$ & $\begin{array}{l}24.82 \\
(11.75 \\
37.89)\end{array}$ & $\begin{array}{l}<.01 \\
0.01\end{array}$ & $\begin{array}{l}24.94 \\
(11.15 \\
38.72)\end{array}$ & $\begin{array}{l}<.01 \\
0.01\end{array}$ & $\begin{array}{l}23.48 \\
(9.59 \\
37.38)\end{array}$ & $\dot{0} 01$ \\
\hline $\begin{array}{l}\text { Maximum strain pressure } \\
(\mathrm{mm} \mathrm{Hg})\end{array}$ & $\begin{array}{l}18.66(5.95 \\
31.37)\end{array}$ & $\begin{array}{l}< \\
0.01\end{array}$ & $\begin{array}{l}15.81(2.72 \\
28.89)\end{array}$ & 0.02 & $\begin{array}{l}15.15 \\
(1.84, \\
28.45)\end{array}$ & 0.03 \\
\hline \multicolumn{7}{|l|}{ Rectal pressure } \\
\hline $\begin{array}{l}\text { Maximum cough pressure } \\
(\mathrm{mm} \mathrm{Hg})\end{array}$ & $\begin{array}{l}24.81(9.79 \\
39.84)\end{array}$ & $\begin{array}{l}< \\
0.01\end{array}$ & $\begin{array}{l}15.75(0.96 \\
30.54)\end{array}$ & 0.04 & $\begin{array}{l}16.95 \\
(1.97 \\
31.93)\end{array}$ & 0.03 \\
\hline $\begin{array}{l}\text { Maximum strain pressure } \\
(\mathrm{mm} \mathrm{Hg})\end{array}$ & $\begin{array}{l}24.68 \\
(13.93 \\
35.43)\end{array}$ & $\begin{array}{l}< \\
0.01\end{array}$ & $\begin{array}{l}20.70(9.67 \\
31.73)\end{array}$ & $\dot{0} 01$ & $\begin{array}{l}21.39 \\
(10.19 \\
32.60)\end{array}$ & $\dot{0} 01$ \\
\hline Defecation index & $\begin{array}{l}-0.01(-0.36 \\
0.34)\end{array}$ & 0.96 & $\begin{array}{l}-0.04(-0.40 \\
0.33)\end{array}$ & 0.84 & $\begin{array}{l}-0.03 \\
(-0.40 \\
0.35)\end{array}$ & 0.89 \\
\hline $\begin{array}{l}\text { Threshold volume needed to } \\
\text { elicit RAIR }(\mathrm{mL})\end{array}$ & $\begin{array}{l}1.39(-2.40 \\
5.19)\end{array}$ & 0.47 & $\begin{array}{l}0.94(-3.07 \\
4.94)\end{array}$ & 0.64 & $\begin{array}{l}1.36(-2.68 \\
5.40)\end{array}$ & 0.51 \\
\hline \multicolumn{7}{|l|}{ Rectal sensation } \\
\hline First sensation (mL) & $\begin{array}{l}1.38(-3.05 \\
5.81)\end{array}$ & 0.54 & $\begin{array}{l}-1.16(-6.30, \\
3.99)\end{array}$ & 0.66 & $\begin{array}{l}0.45(-4.22, \\
5.12)\end{array}$ & 0.85 \\
\hline Desire to defecate $(\mathrm{mL})$ & $\begin{array}{l}-3.55 \\
(-11.77 \\
4.67)\end{array}$ & 0.39 & $\begin{array}{l}-6.51 \\
(-15.72 \\
2.70)\end{array}$ & 0.16 & $\begin{array}{l}-4.20 \\
(-12.94 \\
4.55)\end{array}$ & 0.34 \\
\hline
\end{tabular}




\begin{tabular}{|c|c|c|c|c|c|c|}
\hline & Model 1 & & Model 2 & & Model 3 & \\
\hline Urge to defecate (mL) & $\begin{array}{l}-0.63 \\
(-15.13 \\
13.86)\end{array}$ & 0.93 & $\begin{array}{l}-3.06 \\
(-18.28 \\
12.16)\end{array}$ & 0.69 & $\begin{array}{l}-1.84 \\
(-17.26 \\
13.58)\end{array}$ & 0.81 \\
\hline
\end{tabular}

\section{Discussion}

This is the first study to report normal HRAM values in healthy Vietnamese adults, and predominately includes anorectal pressure and rectal sensation values. In addition, we also measured other values, including functional anal canal length, HPZ length, defecation index, and the threshold volume to elicit RAIR. All HRAM values were compared by sex, age group, and BMI, in which anorectal pressure and rectal sensation values were significantly different between men and women. We found that a significant proportion of healthy people had dyssynergic patterns during simulated defecation while in a prone position. This study provided evidence of normal HRAM values along with the proportion of dyssynergic patterns in healthy adults which may be considered baseline data with which to compare clinical measurements in order to enhance diagnosis and treatment for anorectal diseases in Vietnam, and to allow comparison with future studies.

Our results also show similarities in the patterns and range of values when compared with those of previous studies regarding normal HRAM values in healthy people $[8-11,16]$. However, besides the values for anorectal pressure and rectal sensation, previous studies reported different parameters. Although these studies focused on normal HRAM values, they had specific goals in their reporting and methodology, and so the manner and structure of HRAM indicators presented differed from ours. There are currently no guidelines for the selection of indicators when conducting HRAM, so difficulties in comparing results between studies may arise. As such, we suggest that a standard international HRAM measurement reporting scheme should be developed $[3,11,17]$.

Except for the study conducted by Carrington et al. [11], previous studies did not report functional anal canal length values $[8-10,16]$. The mean value of functional anal canal length was slightly higher in our study than that reported by Carrington et al. [11]. We also did not find a significant difference in this value between males and females. We did find a significant difference in the functional anal canal length between participants with $\mathrm{BMI}<25$ and those with $\mathrm{BMI} \geq 25$, suggesting that $\mathrm{BMI}$ variation could affect the functional anal canal length. In the study conducted by Paul et al., functional anal canal length was used to evaluate anal sphincter dysfunction in patients with fecal incontinence or constipation, using baseline values collected from a healthy volunteer control group [18]. However, functional anal canal length did not help diagnosis of fecal incontinence or constipation in that study. Similarly, in the present study HPZ values were relatively similar to other studies $[9,10,19]$. We also found no difference in HPZ values according to sex and age group. Previously, it has been shown that patients with increased HPZ 
lengths may suffer from defecatory disorders [19]. We found a statistically significant difference in HPZ values according to $B M I$, such that participants with $B M I \geq 25$ had increased HPZ values when compared to those of participants with $\mathrm{BMI}<25$. BMI may as such also affect HPZ values, and we therefore suggest that further studies be conducted to better determine how BMI and other factors influence normal HPZ values.

Anorectal pressure values in our study are similar to those reported in previous studies, but there were certain differences. Resting pressure in our study was similar to that reported by Carrington et al. [11], although our value was higher those reported by Li et al. [9], Lee et al. [8], and Jones et al. [16]. Similar to previous reports, we found no significant difference in anal resting pressure between males and females $[9,11]$. In a study conducted in healthy Korean people, Lee et al. reported that the maximum anal resting pressure was higher in men than in women [8], although that result may be influenced by the relatively small sample size of that study. Maximum anal squeeze pressure results from our study were lower than that of Carrington et al. [11] and Li et al. [9]. However, mean maximum anal squeeze pressure in our study was higher than that of Jones et al. [16]. Differences in values may relate to variation among participants, measuring instruments, or measurement protocol. Furthermore, the interpersonal interaction between clinical technicians and research participants may also affect measurement results [20]. As in previous studies $[8,11]$, we also found a statistically significant difference in maximum anal squeeze pressure between men and women. In addition, we report a number of other values not previously mentioned, including maximum anal cough pressure, maximum anal strain pressure, maximum rectal cough pressure, and maximum rectal strain pressure. These values also tended to be higher in men than in women. We suggest that these are also important values in the evaluation of anorectal functions, and that these values can be used as a reference for the diagnosis and treatment of anorectal disorders in the future.

The defecation index is simple and easy to calculate and is very effective in assessing disorders that are related to rectoanal coordination. This index is often referred to in studies involving certain anorectal diseases $[21,22]$. If we consider a defecation index $<1.3$ as indicative of an anorectal disorder, approximately $50 \%$ of participants in our study displayed such a condition. However, the participants in our study were healthy people, and this is something that should be taken into account when using the defecation index in diagnosis, as its use may give false-positive results. The mean defecation index in our study is similar to that reported by Lee et al. [8]. We also did not find significant differences in defecation index according to gender, age group, or BMI group.

Dyssynergic patterns were defined by Rao et al. to help diagnose patients with chronic constipation [23]. However, most previous studies of HRAM in healthy people did not assess dyssynergic patterns [8-11, 16]. In one study that used HDAM-3D, Enrique et al. reported that a dyssynergic defecation pattern occurred in more than $60 \%$ of health study participants [24]. In our study, approximately $50 \%$ of study participants displayed a dyssynergic pattern, which is consistent with the previously-reported value. We also compared the presence of a dyssynergic pattern by sex, age group, and BMI group, but we did not 
detect any statistically significant differences. Study that includes a larger sample size should be conducted to learn more about these factors.

RAIR is also considered an important indicator in HRAM as it can serve as a proxy signal that associates with various anorectal disorders [25-28]. In our study, RAIR was present in all patients, but the threshold volume to elicit RAIR was different. Although no previous studies have reported threshold volumes that elicited RAIR using HRAM [25-28], we suggest that this is also an appropriate indicator to use as a basis for comparisons of diagnoses. We found that the threshold volume to elicit RAIR was significantly different between the participant age groups, indicating that age might be a factor relating to changes in the structure of the anorectal area. This result is in line with a study that used high-definition anorectal manometry (HDAM-3D) [24], in which Coss-Adame et al. reported that the threshold volume that elicited RAIR was $16.1 \pm 1.4 \mathrm{~mL}$ in a sample of 78 healthy participants, with no significant difference found between males and females. Using the conventional ARM method, it was suggested that RAIR may be absent in adult patients with megacolon, chronic constipation, or chronic intestinal pseudo-obstruction [29-31]. In children, however, the absence of RAIR is considered to be a highly sensitive and highly specific indicator of Hirschsprung disease [32].

With regards to rectal sensation, the results of our study are similar to those reported by Li et al. [9]. We found no significant difference in rectal sensation between men and women. However, as opposed to Li et al., we detected a significant difference in the volumes at first sensation and the threshold of desiring to defecate according to age group [9]. However, in an study using HDAM-3D, Coss-Adame et al. reported associations between sex and values of the threshold of the desire to defecate, the urgency to defecate, and the maximal tolerable volume [24]. In addition, we observed a significant difference in the volume at first sensation according to BMI groups. We suggest that age and BMI may influence the threshold of rectal sensation, although this conflicts with Lee et al., who also reported an association between BMI and the threshold of the first sensation, but one that was negatively correlated [8]. As the mechanisms involved in rectal sensation are still unknown, possible explanations of differences between study outcomes are unclear. A study that includes a larger sample size is needed to better understand the factors related to rectal sensation.

We are aware of some limitations of this study. The sample size was relatively small, and data were stratified according to gender, age, and BMI. The small sample size in a sub-group might thus affect statistical values during comparisons. In this study, we mainly focused on the comparison between males and females, so it may be possible that we missed characteristics that are unique to females, such as any history of maternity-related issues. This is a single-center study, so care must be taken when applying these results to other health centers. Sampling may have been insufficient to cover all possible age groups, and it is possible that patient age may affect HRAM values. In addition, this is a cross-sectional study, so care must be taken when interpreting the results in a causal relationship.

\section{Conclusion}


This study establishes normal values of HRAM in healthy Vietnamese adults, particularly with regards to normal values of anorectal pressure and rectal sensation. We have found that sex is an important factor affecting anorectal pressure in healthy adults. Differences in HRAM values according to age group were only significant for rectal sensations, including first sensation and the desire to defecate. There is almost no difference in HRAM values between BMI groups. Half of study participants had dyssynergic patterns during the simulated strain period, among which types I and III were most prevalent. Further studies that include larger sample sizes should be conducted to further confirm the constants and their relationships, and should focus on specific age groups in order to identify normal values to serve as a reference for diagnosis and treatment.

\section{Abbreviations}

\section{ARM}

Anorectal Manometry

BMI

Body Mass Index

Cl

Confidence Interval

\section{HDAM-3D}

high-definition anorectal manometry

HPZ

High-Pressure Zone

HRAM

High-Resolution Anorectal Manometry

RAIR

Recto-Anal Inhibitory Reflex

SD

Standard Deviation

\section{Declarations}

\section{Ethics approval and consent to participate}

All procedures performed in studies involving human participants were in accordance with the ethical standards of the Hanoi Medical University committee, Vietnam, and with the 1964 Helsinki Declaration and its later amendments or comparable ethical standards. Approval to conduct the study was obtained from the committee of the Hanoi Medical University, Vietnam, by Decision no. 21NCS17/HDDDDHYHN, dated 08/02/2018. All patients gave their written, informed consent prior to inclusion in the study.

\section{Consent for publication}


Not applicable

\section{Availability of data and materials}

The datasets used and/or analyzed during the current study are available from the corresponding author upon reasonable request.

\section{Competing interests}

The authors declare that they have no conflict of interest

\section{Funding}

No funding or sponsorship was received for this study or publication of this article. The article processing charges were funded by the authors.

\section{Authors' contributions}

NNA and VDK- study concept, study design, acquisition of data, statistical analysis, data interpretation, drafting of the manuscript. LDT and NXH: study concept, critical revision of the manuscript. LMC, NXH, NTTH and NVH: data interpretation, critical revision of the manuscript. All authors have read and approved the final manuscript.

\section{Acknowledgements}

The authors thank the healthy volunteers who participated in this study.

\section{References}

1. Azpiroz F, Enck P, Whitehead WE. Anorectal functional testing: review of collective experience. Am J Gastroenterol. 2002;97(2):232-40.

2. Lee TH, Bharucha AE. How to Perform and Interpret a High-resolution Anorectal Manometry Test. J Neurogastroenterol Motil. 2016;22(1):46-59.

3. Rao SS. Pathophysiology of adult fecal incontinence. Gastroenterology. 2004;126(1 Suppl 1):14-22.

4. Lunniss PJ, Gladman MA, Hetzer FH, Williams NS, Scott SM. Risk factors in acquired faecal incontinence. J R Soc Med. 2004;97(3):111-6.

5. Diamant NE, Kamm MA, Wald A, Whitehead WE. AGA technical review on anorectal testing techniques. Gastroenterology. 1999;116(3):735-60.

6. Scott SM, Gladman MA. Manometric, sensorimotor, and neurophysiologic evaluation of anorectal function. Gastroenterol Clin North Am. 2008;37(3):511-38. vii. 
7. Carrington EV, Scott SM, Bharucha A, Mion F, Remes-Troche JM, Malcolm A, Heinrich H, Fox M, Rao SS, International Anorectal Physiology Working G et al: Expert consensus document: Advances in the evaluation of anorectal function. Nat Rev Gastroenterol Hepatol 2018, 15(5):309-323.

8. Lee HJ, Jung KW, Han S, Kim JW, Park SK, Yoon IJ, Koo HS, Seo SY, Yang DH, Kim KJ, et al. Normal values for high-resolution anorectal manometry/topography in a healthy Korean population and the effects of gender and body mass index. Neurogastroenterol Motil. 2014;26(4):529-37.

9. Li Y, Yang X, Xu C, Zhang Y, Zhang X. Normal values and pressure morphology for three-dimensional high-resolution anorectal manometry of asymptomatic adults: a study in 110 subjects. Int $J$ Colorectal Dis. 2013;28(8):1161-8.

10. Noelting J, Ratuapli SK, Bharucha AE, Harvey DM, Ravi K, Zinsmeister AR. Normal values for highresolution anorectal manometry in healthy women: effects of age and significance of rectoanal gradient. Am J Gastroenterol. 2012;107(10):1530-6.

11. Carrington EV, Brokjaer A, Craven H, Zarate N, Horrocks EJ, Palit S, Jackson W, Duthie GS, Knowles $\mathrm{CH}$, Lunniss PJ, et al. Traditional measures of normal anal sphincter function using high-resolution anorectal manometry (HRAM) in 115 healthy volunteers. Neurogastroenterol Motil. 2014;26(5):62535.

12. Cuong LM, Nam V, Ha TT, Ha TT, Hung TQ, Van Loi D, Hung TM, Van Son N, Kien VD. Anorectal Functional Outcomes Following Doppler-Guided Transanal Hemorrhoidal Dearterialization: Evidence from Vietnam. Adv Ther. 2020;37(3):1136-44.

13. Lwanga SK, Lemeshow S, Organization WH. Sample size determination in health studies: a practical manual. 1991.

14. Rao SS, Azpiroz F, Diamant N, Enck P, Tougas G, Wald A. Minimum standards of anorectal manometry. Neurogastroenterol Motil. 2002;14(5):553-9.

15. Rao SS, Patcharatrakul T. Diagnosis and Treatment of Dyssynergic Defecation. J Neurogastroenterol Motil. 2016;22(3):423-35.

16. Jones MP, Post J, Crowell MD. High-resolution manometry in the evaluation of anorectal disorders: a simultaneous comparison with water-perfused manometry. Am J Gastroenterol. 2007;102(4):850-5.

17. Carrington EV, Heinrich $\mathrm{H}$, Knowles $\mathrm{CH}$, Rao SS, Fox M, Scott SM. International Anorectal Physiology Working Party G: Methods of anorectal manometry vary widely in clinical practice: Results from an international survey. Neurogastroenterol Motil. 2017;29(8):e13016.

18. Vollebregt PF, Rasijeff AMP, Pares D, Grossi U, Carrington EV, Knowles CH, Scott SM. Functional anal canal length measurement using high-resolution anorectal manometry to investigate anal sphincter dysfunction in patients with fecal incontinence or constipation. Neurogastroenterol Motil. 2019;31(3):e13532.

19. Ratuapli SK, Bharucha AE, Noelting J, Harvey DM, Zinsmeister AR. Phenotypic identification and classification of functional defecatory disorders using high-resolution anorectal manometry. Gastroenterology. 2013;144(2):314-22 e312. 
20. Heinrich H, Fruehauf H, Sauter M, Steingotter A, Fried M, Schwizer W, Fox M. The effect of standard compared to enhanced instruction and verbal feedback on anorectal manometry measurements. Neurogastroenterol Motil 2013, 25(3):230-7, e163.

21. Seong MK. Assessment of functional defecation disorders using anorectal manometry. Ann Surg Treat Res. 2018;94(6):330-6.

22. Seo M, Joo S, Jung KW, Lee J, Lee HJ, Soh JS, Yoon IJ, Koo HS, Seo SY, Kim D, et al. A high-resolution anorectal manometry parameter based on integrated pressurized volume: A study based on 204 male patients with constipation and 26 controls. Neurogastroenterol Motil. 2018;30(9):e13376.

23. Rao SSC, Azpiroz F, Diamant N, Enck P, Tougas G, Wald A. Minimum standards of anorectal manometry. Neurogastroenterology Motility. 2002;14(5):553-9.

24. Coss-Adame E, Rao SS, Valestin J, Ali-Azamar A, Remes-Troche JM. Accuracy and Reproducibility of High-definition Anorectal Manometry and Pressure Topography Analyses in Healthy Subjects. Clin Gastroenterol Hepatol. 2015;13(6):1143-50 e1141.

25. Kaur G, Gardiner A, Duthie GS. Rectoanal reflex parameters in incontinence and constipation. Diseases of the colon rectum. 2002;45(7):928-33.

26. e Leite ACdA, Moreira MAR, Barbosa MA, Júnior HM, Leite PCCA, Moreira JPT. Clinical and manometric investigation in constipated Chagasic patients with and without megacolon. Journal of Coloproctology. 2019;39(2):145-52.

27. Meinds RJ, Trzpis M, Broens PM. Immaturity of the rectoanal inhibitory reflex as a cause of severe constipation in newborns. Hirschsprung's disease: early diagnosis \& long-term outcomes 2019:68.

28. Thiruppathy K, Mason J, Akbari K, Raeburn A, Emmanuel A. Physiological study of the anorectal reflex in patients with functional anorectal and defecation disorders. Journal of digestive diseases. 2017;18(4):222-8.

29. Basilisco G, Gebbia C, Peracchi M, Velio P, Conte D, Bresolin N, Nobile-Orazio E. Cerebellar degeneration and hearing loss in a patient with idiopathic myenteric ganglionitis. Eur $\mathrm{J}$ Gastroenterol Hepatol. 2005;17(4):449-52.

30. Faussone-Pellegrini MS, Fociani P, Fau - Buffa R, Buffa R, Fau - Basilisco G, Basilisco G. Loss of interstitial cells and a fibromuscular layer on the luminal side of the colonic circular muscle presenting as megacolon in an adult patient. (0017-5749 (Print)).

31. Staller K. Role of Anorectal Manometry in Clinical Practice. Curr Treat Options Gastroenterol. 2015;13(4):418-31.

32. de Lorijn F, Kremer LC, Reitsma JB, Benninga MA. Diagnostic tests in Hirschsprung disease: a systematic review. J Pediatr Gastroenterol Nutr. 2006;42(5):496-505. 